\title{
Changes in extra-striatal functional connectivity in patients with schizophrenia in a psychotic episode
}

Henning Peters, Valentin Riedl, Andrei Manoliu, Martin Scherr, Dirk Schwerthöffer, Claus Zimmer, Hans Förstl, Josef Bäuml, Christian Sorg* and Kathrin Koch*

\section{Background}

In patients with schizophrenia in a psychotic episode, intra-striatal intrinsic connectivity is increased in the putamen but not ventral striatum. Furthermore, multimodal changes have been observed in the anterior insula that interact extensively with the putamen.

\section{Aims}

We hypothesised that during psychosis, putamen extrastriatal functional connectivity is altered with both the anterior insula and areas normally connected with the ventral striatum (i.e. altered functional connectivity distinctiveness of putamen and ventral striatum).

\section{Method}

We acquired resting-state functional magnetic resonance images from 21 patients with schizophrenia in a psychotic episode and 42 controls.

\section{Results}

Patients had decreased functional connectivity: the putamen with right anterior insula and dorsal prefrontal cortex, the ventral striatum with left anterior insula. Decreased functional connectivity between putamen and right anterior insula was specifically associated with patients' hallucinations. Functional connectivity distinctiveness was impaired only for the putamen.

\section{Conclusions}

Results indicate aberrant extra-striatal connectivity during psychosis and a relationship between reduced putamen-right anterior insula connectivity and hallucinations. Data suggest that altered intrinsic connectivity links striatal and insular pathophysiology in psychosis.

\section{Declaration of interest}

None.

\section{Copyright and usage}

(c) The Royal College of Psychiatrists 2017.
The pathophysiology of the striatum is one of the key elements in our understanding of schizophrenia, particularly of psychotic states. ${ }^{1,2}$ Increased striatal dopamine has been suggested as a 'final common pathway to psychosis. ${ }^{2}$ This idea is supported by findings of increased striatal dopamine transmission during prodromal and psychotic states. ${ }^{3,4}$ Levels of hyperdopaminergia correlate with psychosis severity and antidopaminergic drugs reduce psychotic symptoms in most cases. ${ }^{2,5}$ Recent in-vivo positron emission tomography studies indicate that particularly the presynaptic dopamine concentration is increased, mainly in the dorsal striatum. ${ }^{4}$ This regional specificity is further supported by resting-state functional magnetic resonance imaging (rs-fMRI) results, demonstrating that functional connectivity of ongoing activity within the striatum is selectively increased in the putamen. Such intra-striatal functional connectivity changes have been shown to be only present during psychosis and associated with the severity of positive symptoms, whereas functional connectivity changes within the ventral striatum were only found during psychotic remission and linked with negative symptoms. ${ }^{6}$ Because of striatum's involvement in cortico-basal-ganglia-thalamocortical loops, the question arises whether, beyond specifically aberrant intra-striatal connectivity, extra-striatal functional connectivity with cortical regions is also specifically changed, in particular for the putamen.

First, patterns of cortical extra-striatal functional connectivity are distinct for dorsal and ventral striatum. ${ }^{7}$ While ventral striatum functional connectivity includes the ventromedial prefrontal cortex and orbitofrontal cortex, the putamen is mainly

*These authors contributed equally to this work. linked with the anterior insula, anterior cingulate cortex and medial/lateral prefrontal cortex. ${ }^{7,8}$ Previous imaging studies demonstrated changes in extra-striatal connectivity in patients with schizophrenia. ${ }^{6}$ For example, during cognitive tasks such as attentional oddball, decreased frontostriatal functional connectivity was found in patients, with progressive decreases being associated with disorder severity and task duration. ${ }^{9}$ Resting-state studies of ongoing brain activity revealed aberrant functional connectivity from various frontal regions including the anterior cingulate cortex, dorsolateral prefrontal cortex and orbitofrontal cortex with the striatum in prodromal state and patients with psychosis. ${ }^{10-13}$ Most recently, altered striatal functional connectivity with cortical regions has been observed from increased to decreased connectivity along a ventral-dorsal axis within the striatum in patients with first-episode psychosis and their relatives; this result indicates complex and disease risk-related reorganisation of extrastriatal functional connectivity across striatal subregions in schizophrenia. ${ }^{14}$ Particularly across different striatal subsystems, this complex pattern suggests less distinctiveness of functional connectivity for different subregions especially in psychosis. More specifically, accounting for putamen's prominent role in psychosis and its intra-striatal reorganisation, we hypothesised that relative to the ventral striatum - putamen functional connectivity with the cortex might be increased for regions that are normally more strongly connected with the ventral striatum.

Second, from the perspective of intrinsic networks (i.e. consistent spatial patterns of coherent ongoing brain activity), the putamen is intimately associated with the salience network. ${ }^{8}$ The salience network covers insula, anterior cingulate cortex and parts of the dorsomedial and dorsolateral prefrontal cortex. It 
processes emotionally salient stimuli from the body and external world and controls, particularly via its right anterior insula, interactions between other networks such as default mode or central executive network. ${ }^{15}$ Recently, aberrant salience network connectivity and control function was observed in patients in a psychotic episode and associated with hallucinations with aberrations converging on the right anterior insula. ${ }^{16,17}$ These findings were specific for psychosis, since during psychotic remission left rather than right anterior insula connectivity was relevant for aberrant network interactions and patients' negative symptoms. ${ }^{16}$ Together with intra-striatal changes, these data suggest that selective changes of the putamen and right anterior insula in psychosis might be related. Based on these findings, we hypothesised that putamen's extra-striatal functional connectivity with the right anterior insula is specifically altered in patients in a psychotic episode and less distinct relative to the ventral striatum.

To test these hypotheses, we measured blood oxygenation level-dependent (BOLD) activity in 42 healthy controls and 21 patients with schizophrenia in a psychotic episode by use of rs-fMRI. Imaging data of half of the healthy volunteers were used as independent regional priors for subsequent group comparisons between patients and the other half of the controls, to increase specificity of findings. Main outcome measures were individual $\beta$-maps of seed-based functional connectivity applied to putamen and ventral striatum. To estimate group differences, $\beta$-maps were compared across groups via voxelwise two-sample $t$-tests. To investigate the relationship between psychotic symptoms and aberrant extra-striatal functional connectivity in patients, correlation analysis was performed. To analyse distinctiveness of extra-striatal functional connectivity for putamen and ventral striatum, we defined distinct functional connectivity by averaged incongruent seed-target-functional connectivity values (i.e. averaged functional connectivity values of functional connectivity seed in the putamen and functional connectivity target as defined by the functional connectivity map of the ventral striatum and vice versa ${ }^{18}$ ).

\section{Method}

\section{Participants}

In total, 42 healthy controls (control group) and 21 patients with schizophrenia in a psychotic episode (schizophrenia group) participated in the study. All patients and 21 healthy controls had been investigated in a previous study mentioned above, ${ }^{6}$ which investigated intra-striatal functional connectivity changes. For the current study of extra-striatal functional connectivity, an additional 21 group-matched healthy controls were recruited as an independent control group to improve extra-striatal functional connectivity analysis sensitivity and specificity. Written informed consent in accordance with the Human Research Committee guidelines of the Klinikum Rechts der Isar, Technische Universität München was obtained from all participants. Patients were recruited from the Department of Psychiatry, Klinikum Rechts der Isar TU München and controls by word-of-mouth advertising. Participants' examination included medical history, psychiatric interview, psychometric assessment, urine drug screening and additionally blood tests for the schizophrenia group. The global level of social, occupational, and psychological functioning was measured with the Global Assessment of Functioning Scale (GAF). ${ }^{19}$ Psychiatric diagnoses relied on the DSM-IV. ${ }^{20}$ To assess psychiatric diagnoses, the Structured Clinical Interview for DSM-IV (SCID-I) was used. ${ }^{19}$ For rating severity of clinical symptoms on the day of scanning, the Positive and Negative Syndrome Scale (PANSS) was applied. ${ }^{21}$ Clinical psychometric assessment was completed by psychiatrists (D.S. and M.S.) who have been professionally trained for SCID and PANSS-based interviews with interrater reliability of more than $95 \%$.

Inclusion criteria for the study were diagnosis of schizophrenia, acute psychosis, particularly during the fMRI session (at least three positive PANSS subscores $\geqslant 3$ ), and age between 18 and 60 years. Exclusion criteria were current or past neurological or internal systemic disorder, current depressive or manic episode, substance misuse (except for nicotine) and cerebral pathology on MRI.

All the schizophrenia group were diagnosed with paranoid schizophrenia during acute psychosis as indicated by clinical exacerbation and increased positive symptom scores on the PANSS (Table 1). In total, 7 out of 21 had significant hallucinations (PANSS P3 $\geqslant 3$ ), 15 delusions (PANSS P1 $\geqslant 3$ ). The mean duration of illness was 7.15 years $($ s.d. $=6.89)$, the mean number of hospital admissions was 2.98 (s.d. $=2.48$ ). Concerning medication, three patients were free of any antipsychotic medication. All other patients received mono- or dual therapy with atypical antipsychotic medication (see Table 1 and online Table DS1). All patients have been treated previously with antipsychotic drugs (i.e. none of the patients was treatment naive). The control group were all free of any current or past psychiatric, neurological or systemic disorder or psychotropic medication.

Behavioural and imaging data from the schizophrenia group and 21 controls (the 'comparison control group') were used in a previous study, ${ }^{6}$ which focused on intra-striatal functional connectivity. An additional 21 group-matched healthy controls were recruited as an independent control group to improve functional connectivity analysis sensitivity and specificity (see below).

\section{MRI data acquisition}

MRI was carried out using a 3T whole-body MR scanner (Achieva, Philips, The Netherlands) using an eight-channel phased-array head coil. $T_{1}$-weighted anatomical data were obtained by a magnetisation-prepared rapid acquisition gradient echo sequence (echo time $(\mathrm{TE})=4 \mathrm{~ms}$, repetition time $(\mathrm{TR})=9 \mathrm{~ms}$, inversion time $(\mathrm{TI})=100 \mathrm{~ms}$, flip angle $5^{\circ}$, field of view $($ FoV $)=240 \times 240 \mathrm{~mm}$, matrix $240 \times 240,170$ slices, voxel size $1 \times 1 \times 1 \mathrm{~mm})$. Data from rs-fMRI were obtained by a gradientecho echo-planar imaging (EPI) sequence ( $\mathrm{TE}=35 \mathrm{~ms}$, $\mathrm{TR}=2000 \mathrm{~ms}$, flip angle $82^{\circ}, \quad \mathrm{FoV}=220 \times 220 \mathrm{~mm}$, matrix $80 \times 80$, 32 slices, slice thickness $4 \mathrm{~mm}$, and $0 \mathrm{~mm}$ interslice gap). All participants underwent $10 \mathrm{~min}$ of rs-fMRI resulting in 300 volumes. As in most previous rs-fMRI studies (e.g. Di Martino et $a l^{7}$ Seeley $e t a l^{8}$ ), we instructed participants to keep their eyes closed and not to fall asleep. We verified that participants stayed awake by interrogating via intercom immediately after the rs-fMRI scan.

\section{MRI data analysis}

\section{Preprocessing}

For each participant, the first three rs-fMRI scans were discarded because of magnetisation effects. SPM8 (Wellcome Department of Cognitive Neurology, London) was used for motion correction, spatial normalisation into the stereotactic space of the Montreal Neurological Institute (MNI) and spatial smoothing with an $8 \times 8 \times 8 \mathrm{~mm}$ Gaussian kernel. To ensure data quality, particularly concerning motion-induced artefacts, temporal signal-to-noise ratio (tSNR) and point-to-point head motion were estimated for each participant. ${ }^{22,23}$ Point-to-point motion was defined as the absolute displacement of each brain volume compared with its previous volume. Moreover, root mean square (RMS) of the translational head movement parameters was calculated for each 


\begin{tabular}{|c|c|c|c|c|c|}
\hline Measure & $\begin{array}{l}\text { Independent } \\
\text { control group } \\
\quad(n=21)\end{array}$ & $\begin{array}{l}\text { Comparison } \\
\text { control group } \\
\quad(n=21)\end{array}$ & $\begin{array}{l}\text { Schizophrenia } \\
\text { group }(n=21)\end{array}$ & \multicolumn{2}{|c|}{$\begin{array}{l}\text { Schizophrenia group }(n=21) \mathrm{v} \text {. } \\
\text { comparison control group }(n=21)^{2}\end{array}$} \\
\hline Age, mean (s.d.) & $33.49(12.9)$ & $33.57(13.6)$ & $34.05(12.27)$ & -0.121 & 0.904 \\
\hline Gender (men/women), $n$ & $10 / 11$ & $10 / 11$ & $10 / 11$ & & \\
\hline \multicolumn{6}{|l|}{ Positive and Negative Syndrome Scale, mean (s.d.) } \\
\hline Total & - & $30.14(0.65)$ & $80.76(20.77)$ & 8.96 & $<0.001 *$ \\
\hline Positive & - & $7.05(0.22)$ & $19.4(6.09)$ & 9.091 & $<0.001 *$ \\
\hline Negative & - & $7.10(0.44)$ & $21.14(8.20)$ & 7.84 & $<0.001^{*}$ \\
\hline General & - & $16.05(0.22)$ & $39.81(11.06)$ & 9.846 & $<0.001^{*}$ \\
\hline Global Assessment of Functioning Scale, mean (s.d.) & - & $99.76(1.09)$ & $39.62(11.68)$ & -23.492 & $<0.001^{*}$ \\
\hline Chlorpromazine-equivalent dose, mean (s.d.) & - & & $388.61(384.67)$ & & \\
\hline
\end{tabular}

participant. ${ }^{23}$ Excessive head motion (cumulative motion translation $>3 \mathrm{~mm}$ and mean point-to-point translation or rotation $>0.15 \mathrm{~mm}$ or 0.1 ) was applied as exclusion criterion. None of the participants had to be excluded. Two-sample $t$-tests yielded no significant differences between groups regarding mean pointto-point translation or rotation of any direction $(P>0.15)$, RMS $(P>0.2)$, or tSNR $(P>0.40)$. Further control for head motion effects was carried out in the individual-level functional connectivity analysis.

\section{Individual-level functional connectivity analysis}

Seeds of functional connectivity analysis were selected according to coordinates of group-different regions derived from our first study in patients during acute psychosis (putamen) and after remission (ventral striatum) relative to healthy controls. ${ }^{6}$ We created spherical regions of interest (ROIs, $6 \mathrm{~mm}$ radius) for the dorsal striatum, i.e. left and right putamen $(+/-24,12,0)$ and the ventral striatum, i.e. nucleus accumbens $(+/-12,9,-9)$, respectively, by the use of MarsBaR (v0.42, http://marsbar. sourceforge.net/). Centres of ROIs were derived from the study of Martinez and colleagues and corresponded to centres in our previous study. ${ }^{6,24}$

After Butterworth bandpass-filtering of all voxel time courses for the frequency range from 0.009 to $0.08 \mathrm{~Hz}$, we extracted voxel time courses of seed ROIs and reduced them to ROI-representative time courses by singular value decomposition. Each time course was entered into a first-level fixed-effects general linear model in SPM8, and four separate functional connectivity analyses (i.e. left/right ventral striatum, left/right putamen) were performed for each participant yielding four functional connectivity maps for subsequent second-level analyses. Regressors for global grey matter, white matter, cerebrospinal fluid (CSF) BOLD-signal, and six movement parameters for each participant were included as covariates of no interest in each model. ${ }^{7}$ As the global grey matter signal is thought to reflect a combination of physiological processes (such as cardiac and respiratory fluctuations) and scanner drift, it was included as a nuisance signal to minimise the influence of such factors. ${ }^{25}$ To extract the nuisance covariate time series for grey matter, white matter and CSF, each individual's high-resolution $T_{1}$-weighted structural image was segmented. Mean images of study sample's $T_{1}$-segmentation were used to create ROIs for the extraction of grey matter, white matter and CSF nuisance signals.

\section{Group-level functional connectivity analysis}

Group analyses were performed using $\beta$-maps from individuallevel functional connectivity analysis in separate flexible factorial models of analysis of variance (ANOVA). More specifically, all
ANOVA models included covariates of no interest (gender, age, seed regional volumes of ventral striatum and putamen, respectively; see voxel-based morphometry (VBM) analysis below) and were restricted to explicit masks of ventral striatum and putamen functional connectivity, respectively. Masks were created by use of a flexible factorial ANOVA model of putamen/ventral striatum functional connectivity images of the independent control group (factors: hemisphere with levels left/right and seed-ROI with levels ventral striatum/putamen); appropriate post-hoc t-tests revealed positively correlated functional connectivity maps for the ventral striatum and putamen, respectively $(P<0.05$ uncorrected for masks; $P<0.05$ family-wise error (FWE)-corrected on voxel level for online Fig. DS1). Then, to analyse group differences of putamen functional connectivity, a flexible factorial ANOVA $(P<0.05$ FWE-corrected on voxel level and restricted to a mask of the independent control group) was applied to putamen functional connectivity images of currently symptomatic patients and the comparison control group with factors group (levels: patient/ control) and seed-ROI (levels: left/right putamen). Analogous ANOVA was applied for ventral striatum functional connectivity. For both ANOVA models, the main effect of group (and corresponding post-hoc t-tests to reveal direction of change) was the effect of interest. Reported voxel coordinates correspond to standardised MNI space. To visualise results, we used MRIcroN (http://www.nitrc.org/projects/mricron).

\section{Brain-behaviour relationship}

To investigate the relationship between striatal functional connectivity differences and psychotic symptoms, $\beta$-values of regional group differences in putamen and ventral striatum functional connectivity for each cluster and patient were averaged across voxels and entered into partial correlation analyses. According to previous results, we chose subscores instead of the summed positive symptom score. Since only hallucinations and delusions correlated with striatal functional connectivity decreases, we limited analyses to these subscores. ${ }^{6}$ Covariates of no interest were age and gender as well as medication levels reflected by chlorpromazine-equivalent units $(\mathrm{CPZ}){ }^{26}$ The significance threshold was set to $P<0.007$, Bonferroni-corrected for seven group-different clusters of functional connectivity decreases (the seven clusters are listed in Table 2).

\section{Distinct functional connectivity}

We expected that in the schizophrenia group, putamen's functional connectivity is changed both with the anterior insula and with areas that are typically connected more strongly with the ventral striatum. To examine such altered distinctiveness of putamen and ventral striatum connectivity, we defined distinct 


\begin{tabular}{|c|c|c|c|c|c|c|}
\hline Seed & Anatomical regions & Side & $\begin{array}{l}\text { Cluster size } \\
\text { in voxels, } k\end{array}$ & $\begin{array}{l}\text { MNI, peak voxel } \\
\text { coordinates, } x, y, z\end{array}$ & $z$-score & $P^{a}$ \\
\hline \multirow{6}{*}{ Putamen } & $\begin{array}{l}\text { Inferior frontal gyrus (opercular, orbital) } \\
\text { Anterior insula }\end{array}$ & Right & 236 & $45,15,-6$ & 5.21 & $<0.0001$ \\
\hline & \multirow{5}{*}{$\begin{array}{l}\text { Superior frontal gyrus } \\
\text { Inferior frontal gyrus (triangular) } \\
\text { Middle frontal gyrus } \\
\text { Middle frontal gyrus } \\
\text { Middle frontal gyrus } \\
\text { Middle cingulate cortex }\end{array}$} & Right & 87 & $51,36,6$ & 4.57 & 0.027 \\
\hline & & Right & 61 & $12,12,48$ & 5.50 & $<0.0001$ \\
\hline & & Right & 48 & $36,48,30$ & 4.16 & 0.044 \\
\hline & & Left & 36 & $-36,48,27$ & 4.27 & 0.029 \\
\hline & & Right & 20 & $9,15,39$ & 4.33 & 0.023 \\
\hline $\begin{array}{l}\text { Ventral } \\
\text { striatum }\end{array}$ & $\begin{array}{l}\text { Anterior insula } \\
\text { Inferior frontal gyrus (orbital) }\end{array}$ & Left & 26 & $-36,21,-9$ & 4.37 & 0.011 \\
\hline
\end{tabular}

functional connectivity in terms of averaged incongruent seedtarget functional connectivity values of striatal seeds. For example, the connectivity of the putamen with regions that were normally coupled more strongly with the ventral striatum than the putamen is referred to as incongruent seed-target connectivity of the putamen. By the use of appropriate post-hoc t-tests for the above-mentioned ANOVA model (factors seed (putamen/ventral striatum) and side (left/right) of the independent control group) $(P<0.05$ FWE-corrected), we first obtained distinct functional connectivity maps of the putamen (main effect seed putamen $>$ ventral striatum) and ventral striatum (main-effect seed ventral striatum $>$ putamen $).{ }^{18}$ We used these distinct functional connectivity maps as masks to calculate averaged incongruent seed-target functional connectivity values (for example, for a given participant and the putamen as seed, $\beta$-values of putamen functional connectivity map were averaged across all voxels of the ventral striatum-distinct functional connectivity mask). Incongruent seed-target-functional connectivity values reflect distinctiveness of striatal functional connectivity and were compared across the schizophrenia and comparison control group by use of two-sample $t$-tests $(P<0.05)$. Accordingly, increased incongruent seed-target connectivity for a striatal seed would be interpreted as reduced distinctiveness of striatal functional connectivity because of an enlarged functional connectivity for this seed.

\section{Voxel-based morphometry}

To control for effects of striatal structure on functional connectivity results, we included ventral striatum and putamen volumes as covariates of no interest into statistical models of group comparisons. We used previous results of VBM analysis in the schizophrenia group and the comparison control group as described in the online supplement DS1 and elsewhere. ${ }^{6}$ Briefly, VBM volumes of patients' left and right putamen and ventral striatum were not different from those of the comparison control group.

\section{Results}

In the independent control group, significant functional connectivity was found for the putamen with bilateral inferior, middle, and superior frontal gyrus, anterior insula, anterior and middle cingulate cortex, pallidum and caudate nucleus, for the ventral striatum with orbital parts of the inferior frontal gyrus, the medial superior frontal gyrus, anterior insula, anterior cingulate cortex and pallidum $(P<0.05$ FWE-corrected, online Fig. DS1, Table DS2). Putamen and ventral striatum functional connectivity maps of the schizophrenia group and the comparison control group were largely consistent with these patterns, indicating that the basic pattern of putamen and ventral striatum functional connectivity is preserved in patients (online Fig. DS1).

Group comparisons, which were masked by functional connectivity patterns of the independent control group, revealed that in the schizophrenia group putamen functional connectivity was decreased for bilateral middle frontal gyrus and superior frontal gyrus, the right opercular and triangular part of the inferior frontal gyrus, right anterior insula and right middle cingulate cortex $(P<0.05$ FWE-corrected, online Fig. DS2, Table $2)$. The schizophrenia group's ventral striatum functional connectivity was decreased with the left anterior insula, extending to the orbital part of the left inferior frontal gyrus $(P<0.05$ FWE-corrected, online Fig. DS2, Table 2).

The partial correlation analyses for the patients' decreased regional functional connectivity values and psychotic symptoms $(P<0.007$, Bonferroni-corrected for multiple testing because of seven group-different clusters) showed the decreased functional connectivity between putamen and right anterior insula to be negatively correlated with hallucinations $(P<0.001$, Fig. 1$)$. Neither delusions nor other regions' group-different functional connectivity showed significant results.

To investigate distinctiveness of striatal functional connectivity for putamen and ventral striatum, averaged incongruent seedtarget functional connectivity was calculated for putamen and ventral striatum for each participant. In the schizophrenia group only, the putamen showed significantly increased incongruent seed-target functional connectivity values relative to ventral striatum and different to those of the control group (one- and two-sample $t$-test, $P<0.05$, Fig. 2).

\section{Discussion}

\section{Main findings}

Extra-striatal cortical functional connectivity of the putamen and ventral striatum was studied in patients with schizophrenia during psychosis and in healthy controls using rs-fMRI and seed-based functional connectivity analysis. In patients, putamen's functional connectivity was reduced with the right anterior insula and the dorsomedial and dorsolateral prefrontal cortex, whereas ventral striatum's functional connectivity was decreased with the left anterior insula. Only putamen's aberrant functional connectivity with the right anterior insula was significantly associated with patients' hallucinations. Putamen's functional connectivity in patients was increased with areas regularly connected with the ventral striatum, indicating specifically less distinctive functional connectivity of the putamen relative to the ventral striatum. Data provide evidence that aberrant extra-striatal cortical functional connectivity during psychosis is centred on the putamen, with 


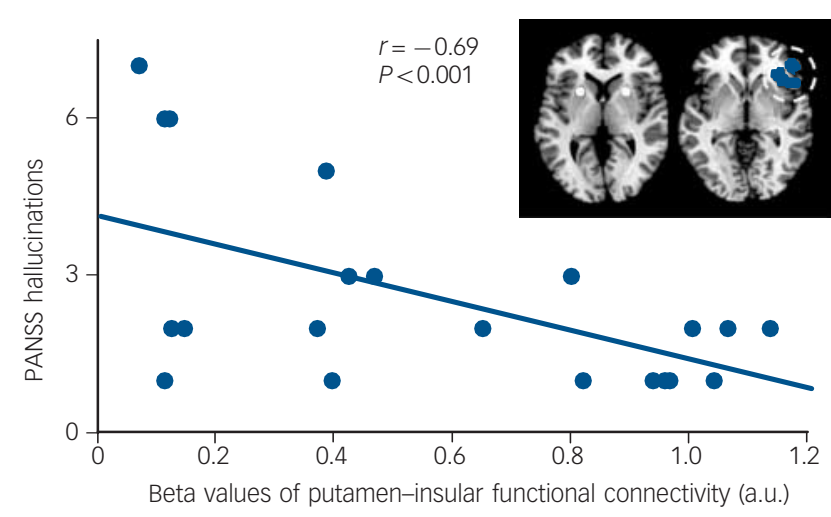

Fig. 1 Negative correlation between hallucinations and functional connectivity between putamen and right anterior insula.

Partial correlation analyses on averaged beta values of functional connectivity group different clusters in online Fig. DS2 with hallucinations and delusion score on the Positive and Negative Syndrome Scale (PANSS) revealed only for the functional connectivity between putamen and right anterior insula a significant relationship with hallucinations (partial correlation coefficient $r=-0,69, P<0.001$, Bonferroni-corrected for multiple testing (i.e. number of group-different clusters). To control for confounding effects, medication levels (chlorpromazine-equivalent dose units), age and gender were included in partial correlation models. a.u., arbitrary units.

both less distinct connectivity focused on the putamen and decreased connectivity between putamen and right anterior insula specifically related to psychotic symptoms. Particularly the last finding suggests aberrant functional connectivity to link striatal and insular pathophysiology in psychosis.

\section{Comparison with findings from other studies}

In the schizophrenia group, we found reduced functional connectivity for both putamen and ventral striatum with cortical regions (online Fig. DS2, Table 2). Putamen functional connectivity was reduced in right anterior insula and dorsomedial and dorsolateral prefrontal cortex, whereas ventral striatum functional connectivity was decreased with the left anterior insula. Group differences were independent of age, gender and striatal volume effects, for which we controlled statistically. Spatial putamen and ventral striatum functional connectivity maps of those in the schizophrenia group were largely comparable with those of the control group, indicating that basic extra-striatal functional connectivity is preserved in schizophrenia (online Fig. DS1). Reduced functional connectivity between putamen and dorsal prefrontal cortex is in line with previous findings. ${ }^{1-14}$ For example, Zhou and colleagues ${ }^{11,12}$ found reduced middle frontal gyrus functional connectivity with the dorsal striatum and Fornito and colleagues ${ }^{14}$ recently observed reduced functional connectivity between the dorsocaudal putamen and dorsal prefrontal cortex in patients with first-episode psychosis, with striatal seed coordinates very near to those of our study. Furthermore, for the same prefrontal cortex areas, authors reported that putamen functional connectivity was also reduced in unaffected first-degree relatives of patients with schizophrenia, suggesting that these connectivity changes may express a specific disease risk more associated with disease trait than state-dependent symptoms. Our finding that reduced functional connectivity between putamen and dorsal prefrontal cortex is not related to psychotic symptoms, supports this suggestion.

On the other hand, we found reduced functional connectivity with right and left anterior insula in patients for both putamen and ventral striatum (online Fig. DS2). This finding is in line with previous findings of reduced striatoinsular functional connectivity in patients in prodromal psychotic states and psychosis. ${ }^{14,27}$ More specifically, Orliac and colleagues recently reported reduced striatal functional connectivity within the salience network centred on anterior insula in patients with schizophrenia. ${ }^{28}$ It should be noted, however, that in the study by Orliac et al, the salience network included the bilateral anterior insula whereas we identified reduced connectivity between the putamen and the right anterior insula in association with reduced connectivity between the ventral striatum and the left anterior insula. The salience network and especially the anterior insula is crucially involved in processing salient internal/external stimuli and controlling interactions between two distinct core cognitive networks, namely the default mode network - involved in selfreferential cognition - and the central executive network involved in goal-driven tasks. ${ }^{8}$ In patients with schizophrenia, the anterior insula is characterised by various structural and functional alterations such as atrophy, impaired white matter structure and aberrant functional connectivity. In particular aberrant functional connectivity of the anterior insula within the salience network is associated with aberrant default-mode network/central executive network interactions and the severity of patients' symptoms. ${ }^{16,29}$ Our finding of reduced functional connectivity between striatum and anterior insula indicates that a disrupted connectivity between striatum and central components of the salience network may constitute a core mechanism underlying the pathophysiology of schizophrenia.

Concerning laterality of aberrant striatal functional connectivity, patients' functional connectivity was reduced for the putamen with the right anterior insula and for the ventral striatum with left anterior insula. Previously we found that only right anterior insula functional connectivity within the salience network was associated with positive symptoms during psychosis, ${ }^{17}$ whereas during remission, left anterior insula connectivity was specifically linked with negative symptoms. ${ }^{29}$ Correspondingly, for the striatum we found that during psychosis, only putamen intra-striatal functional connectivity was increased and linked with positive symptoms, whereas during remission, only ventral striatum functional connectivity was increased and linked with negative symptoms. ${ }^{6}$ Together, it seems that putamen and right anterior insula connectivity is related more with psychosis and positive symptoms, whereas ventral striatum and left anterior insula is related more with remission and negative symptoms. Since the control of vegetative nervous system activity is asymmetrically represented in the anterior insula with sympathetic parts more related to the right anterior insula whereas parasympathetic parts relate more with the left anterior insula, ${ }^{30}$ one might speculate whether lateralised striatal functional connectivity changes in putamen and ventral striatum in psychosis reflect this rather basic asymmetry. Future studies are necessary to investigate such a potential link.

\section{Psychosis and putamen functional connectivity}

With respect to psychosis, two additional findings specify decreased extra-striatal functional connectivity in schizophrenia: (a) aberrant functional connectivity seems to be centred on the putamen relative to the ventral striatum and (b) putamen's decreased functional connectivity with right anterior insula is specifically relevant for psychotic symptoms.

\section{Abnormal distinctiveness of dorsal striatum functional connectivity}

We found less distinct functional connectivity of the striatum in the form of increased putamen functional connectivity with regions regularly connected with the ventral striatum (Fig. 2). This finding suggests that in patients, areas which are preferentially linked with the ventral striatum, show on average increased functional connectivity with the putamen. This finding is focused on the putamen, as distinct functional connectivity of the ventral striatum relative to the putamen was normal in patients (Fig. 2). 
This spatial focus on the dorsal striatum in psychosis corresponds well with previous findings concerning other aspects of striatal pathophysiology. For example, intra-striatal functional connectivity is selectively increased in the putamen during psychosis, and presynaptic dopamine activity is increased during prodromal and psychotic states, especially in the dorsal striatum. ${ }^{1,3,4,6}$ Since such relative dominance of the dorsal striatum is characteristic for habit-like behaviour, some theoretical accounts suggested that patients' pronounced changes in the dorsal striatum might point at a habit-like nature of psychotic symptoms. ${ }^{31,32}$

\section{Aberrant putamen functional connectivity and severity of hallucinations}

In line with this relative dominance of dorsal striatum changes in psychosis, we found explicit evidence that only patients' decreased putamen functional connectivity with the right anterior insula is significantly linked with the degree of hallucinations (Fig. 1). Fornito and colleagues ${ }^{14}$ argue that the previously reported relationship between reduced dorsal striatum functional connectivity and symptom severity may constitute a stateindependent risk marker. However, they mainly discussed functional connectivity with dorsolateral and medial prefrontal cortex and did not consider PANSS subscores. Our finding fit with previous results very well: first, aberrant right anterior insula functional connectivity within the salience network is specifically associated with psychosis, particularly with hallucinations; second, putamen's intra-striatal functional connectivity is selectively associated with psychosis and the degree of psychotic symptoms. ${ }^{6}$ The current result provides a link between these previous findings by extending isolated findings centred on either anterior insula or the dorsal striatum and showing that disrupted functional connectivity between putamen and right anterior insula is specifically related to psychotic key symptoms.

\section{Link between proximal salience, motivational salience and dopamine}

More generally, these data suggest a link between two relevant models of psychosis in schizophrenia, namely the proximal salience model of Palaniyappan ${ }^{17,33}$ and the motivational salience model of Kapur. ${ }^{34,35}$ Although these two concepts integrate a lot of data that are critical for explaining psychotic symptoms in schizophrenia, they emphasise distinct neurocognitive mechanisms centred on distinct key regions, namely the anterior insula and the striatum. More specifically, the concept of proximal salience describes a momentary interoceptive state resulting from the appraisal of external and internal stimuli, which modulates both succeeding learning processes and the selection of actions/ cognitions to improve future evaluation. ${ }^{17}$ In contrast, the concept of motivational salience describes the assignment of a specific motivational value to an internal or external stimulus following its appraisal based on reward prediction error processes. ${ }^{34,35}$ In a neurobiological context, proximal salience has been proposed to be mediated by the anterior insula within the salience network, particularly via modulation of the interaction between other intrinsic connectivity networks, such as the default mode network and central executive network, whereas motivational salience depends highly on striatal activity, which, in turn, is strongly controlled by striatal dopaminergic activity. ${ }^{31,36}$ Latest studies provide strong evidence that psychosis in schizophrenia is characterised by altered functional connectivity within the dorsal striatum as well as in the right anterior insula within the salience network and by aberrant functional connectivity between intrinsic brain networks. ${ }^{16,37}$ However, a direct link between these findings is still missing. Recently, Cole and colleagues ${ }^{38}$ demonstrated that striatoinsular functional connectivity is influenced by pharmacological modulation of striatal dopamine levels. Moreover, Lui et $a l^{39}$ reported increased functional connectivity between the dorsal striatum and the bilateral prefrontal cortex, the parietal cortex and the left superior temporal cortex after short-term treatment with second-generation antipsychotic medication in patients with first-episode schizophrenia. Of note, the increase in functional connectivity was associated with a reduction of clinical symptoms implicating that a pharmacologically induced alteration in functional connectivity leads to significant clinical improvement.

Future studies are required that focus explicitly on the linking potential of striatoinsular intrinsic connectivity between

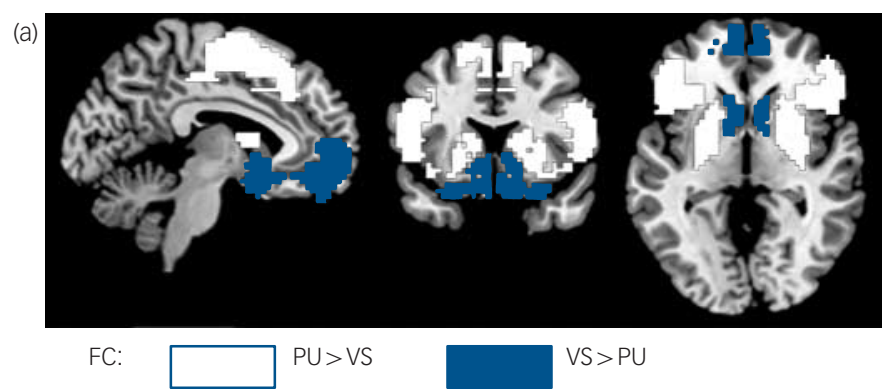

(b)
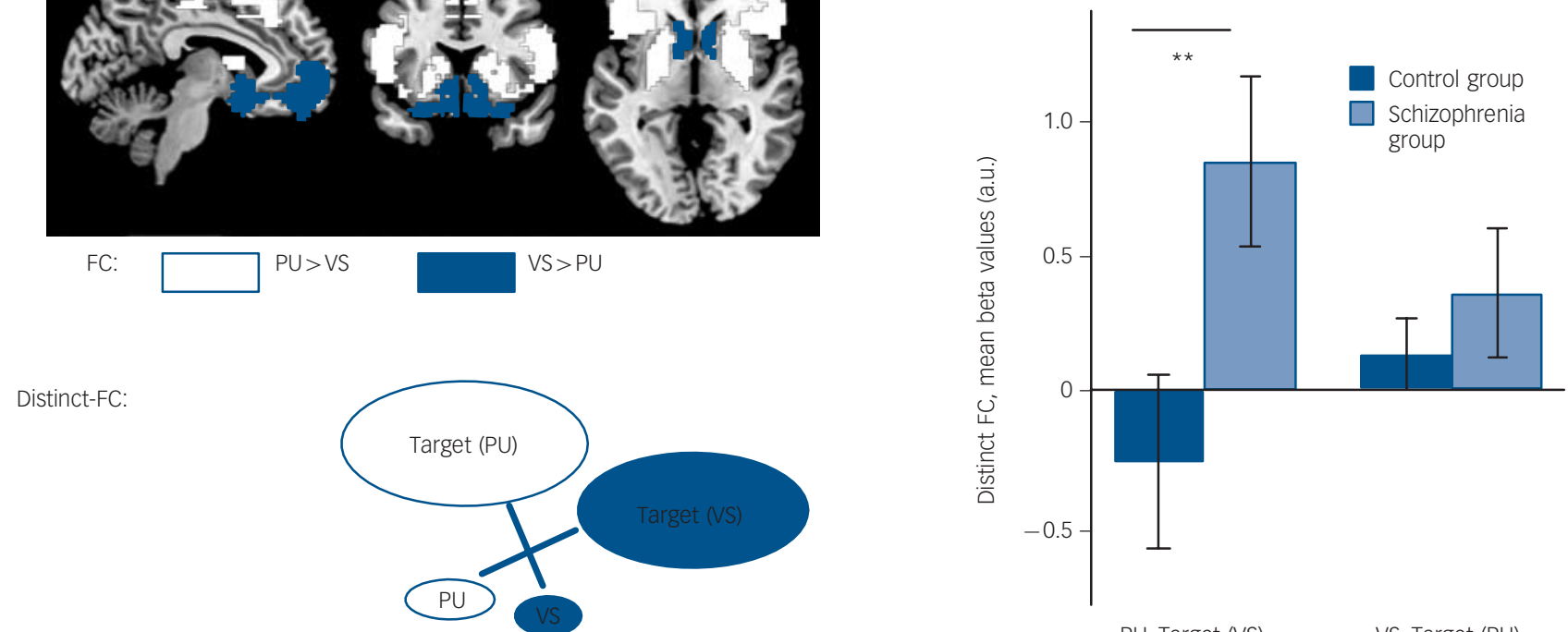

PU-Target (VS)

VS-Target (PU)

Fig. 2 Distinctiveness of functional connectivity (FC) of putamen (PU) and ventral striatum (VS).

(a) White and blue maps represent binary spatial connectivity maps of the independent control group based on post-hoc $t$-tests (putamen $<$ ventral striatum, putamen $>$ ventral striatum) of ANOVA with factors seed (putamen, ventral striatum) and seed side (left, right), $P_{\text {fwE }}<0.05$ corrected for family-wise error (FWE)). The idea of distinct functional connectivity is based on averaged incongruent seed-target functional connectivity for example averaged functional connectivity of the putamen in white with the regular target of the ventral striatum in blue. (b) Mean beta values for distinct functional connectivity for the schizophrenia and the control group are presented. **Indicates significant group differences in distinct functional connectivity (two-sample $t$-tests $P<0.05$ ). a.u., arbitrary units. 
dopaminergic reward prediction error activity in the dorsal striatum, right anterior insula control function on interacting intrinsic networks and psychotic symptoms.

\section{Methodological issues and limitations}

The fact that almost all of the schizophrenia group in our study were medicated may limit the explanatory power of our findings. An influence of antipsychotic medication on functional brain connectivity has been shown. ${ }^{39,40}$ However, a previous comparison between patient subsamples receiving antipsychotics and those who were medication-free did not yield a significant difference in striatal functional connectivity. ${ }^{14}$ To control for the potential confound of medication, we included - with respect to the analysis of the relationship between aberrant functional connectivity and psychotic symptoms - CPZ units in the statistical model of patients' functional connectivity, which did not yield a significant explanatory effect of functional connectivity in the present study. ${ }^{6,16}$ Nevertheless, antipsychotic medication has to be considered when evaluating the current study's results. Future studies in patients who are drug-free might be helpful; however studies in patients who are drug-free but in a psychotic episode might have strong practical and ethical problems.

In conclusion, our results provide evidence that in patients with schizophrenia in a psychotic episode, striatal functional connectivity with the frontoinsular cortex is decreased. Changes were pronounced for the putamen relative to the ventral striatum and decreased connectivity between putamen and right anterior insula was specifically related to psychotic symptoms. Data suggest a link between striatal and anterior insular pathophysiology of psychosis via functional connectivity.

\footnotetext{
Henning Peters, MD, PhD, Department of Psychiatry and TUM-Neuroimaging Cente Klinikum rechts der Isar, Technische Universität München, Munich, Germany; Valentin Riedl, MD, PhD, Department of Psychiatry, Department of Neuroradiology, Department of Nuclear Medicine and TUM-Neuroimaging Center Klinikum rechts de Isar, Technische Universität München, Munich, Germany; Andrei Manoliu, MD, Department of Neuroradiology and TUM-Neuroimaging Center Klinikum rechts der Isar, Technische Universität München, Munich, Germany and Department of Radiology, University Hospital Zürich, Rämistrasse 100, 8091 Zürich, Switzerland: Martin Scherr, MD, Dirk Schwerthöffer, MD, Department of Psychiatry, Technische Universität München, Munich, Germany: Claus Zimmer MD, Department of Neuroradiology, Technische Universität München, Munich, Germany; Hans Förstl MD, Josef Bäuml, MD, Department of Psychiatry, Technische Universität München, MD, Josef Bäuml, MD, Department of Psychiatry, Technische Universität München,
Munich, Germany; Christian Sorg, MD, Department of Psychiatry, Department of Neuroradiology, Department of Nuclear Medicine and TUM-Neuroimaging Center Klinikum rechts der Isar, Technische Universität München, Munich, Germany; Kathrin Koch, PhD, Department of Neuroradiology and TUM-Neuroimaging Center Klinikum rechts der Isar, Technische Universität München, Munich, Germany

Correspondence: Kathrin Koch, Department of Neuroradiology and TUMNeuroimaging Center Klinikum rechts der Isar, Technische Universität München, Ismaninger strasse 22, 81675 Munich, Germany. Email: kathrin.koch@tum.de

First received 26 May 2014, final revision 15 Dec 2014, accepted 27 May 2015
}

\section{Funding}

This work was supported by the German Federal Ministry of Education and Research (BMBF 01ER0803 to C.S.) the Alzheimer Forschung Initiative (AFI 08860 to V.R.) and the Kommission für Klinische Forschung of the Klinikum Rechts der Isar der Technischen Universität München (KKF 8765162 to C.S). We are grateful to the participants of the study and the staff of the Department of Psychiatry and Neuroradiology for their help in recruitment and data collection.

\section{References}

1 Howes OD, Egerton A, Allan V, McGuire P, Stokes P, Kapur S. Mechanisms underlying psychosis and antipsychotic treatment response in schizophrenia: insights from PET and SPECT imaging. Curr Pharm Des 2009; 15: 2550-9.

2 Howes OD, Kapur S. The dopamine hypothesis of schizophrenia: version III - the final common pathway. Schizophr Bull 2009; 35: 549-62.
3 Howes OD, Montgomery AJ, Asselin MC, Murray RM, Valli I, Tabraham P, et al. Elevated striatal dopamine function linked to prodromal signs of schizophrenia. Arch Gen Psychiatry 2009; 66: 13-20.

4 Kegeles LS, Abi-Dargham A, Frankle WG, Gil R, Cooper TB, Slifstein M, et al. Increased synaptic dopamine function in associative regions of the striatum in schizophrenia. Arch Gen Psychiatry 2010; 67: 231-9.

5 Agid O, Mamo D, Ginovart N, Vitcu I, Wilson AA, Zipursky RB, et al. Striatal vs extrastriatal dopamine D2 receptors in antipsychotic response - a doubleblind PET study in schizophrenia. Neuropsychopharmacology 2007; 32 1209-15.

6 Sorg C, Manoliu A, Neufang S, Myers N, Peters H, Schwerthoffer D, et al. Increased intrinsic brain activity in the striatum reflects symptom dimensions in schizophrenia. Schizophr Bull 2013; 39: 387-95.

7 Di Martino A, Scheres A, Margulies DS, Kelly AM, Uddin LQ, Shehzad Z, et al. Functional connectivity of human striatum: a resting state FMRI study. Cereb Cortex 2008; 18: 2735-47

8 Seeley WW, Menon V, Schatzberg AF, Keller J, Glover GH, Kenna H, et al. Dissociable intrinsic connectivity networks for salience processing and executive control. J Neurosci 2007; 27: 2349-56.

9 Morey RA, Inan S, Mitchell TV, Perkins DO, Lieberman JA, Belger A. Imaging frontostriatal function in ultra-high-risk, early, and chronic schizophrenia during executive processing. Arch Gen Psychiatry 2005; 62: 254-62.

10 Salvador R, Martinez A, Pomarol-Clotet E, Sarro S, Suckling J, Bullmore E. Frequency based mutual information measures between clusters of brain regions in functional magnetic resonance imaging. Neuroimage 2007; 35 83-8.

11 Zhou $Y$, Liang $M$, Tian L, Wang $K$, Hao $Y$, Liu $H$, et al. Functional disintegration in paranoid schizophrenia using resting-state fMRI. Schizophr Res 2007; 97 194-205.

12 Zhou Y, Liang M, Jiang T, Tian L, Liu Y, Liu Z, et al. Functional dysconnectivity of the dorsolateral prefrontal cortex in first-episode schizophrenia using resting-state fMRI. Neurosci Lett 2007; 417: 297-302.

13 Tu PC, Lee YC, Chen YS, Li CT, Su TP. Schizophrenia and the brain's control network: aberrant within- and between-network connectivity of the frontoparietal network in schizophrenia. Schizophr Res 2013; 147: 339-47.

14 Fornito A, Harrison BJ, Goodby E, Dean A, Ooi C, Nathan PJ, et al. Functional dysconnectivity of corticostriatal circuitry as a risk phenotype for psychosis. JAMA Psychiatry 2013; 70: 1143-51.

15 Menon V, Uddin LQ. Saliency, switching, attention and control: a network model of insula function. Brain Struct Funct 2010; 214: 655-67.

16 Manoliu A, Riedl V, Zherdin A, Muhlau M, Schwerthoffer D, Scherr M, et al. Aberrant dependence of default mode/central executive network interactions on anterior insular salience network activity in schizophrenia. Schizophr Bull 2014; 40: 428-37.

17 Palaniyappan L, Liddle PF. Does the salience network play a cardinal role in psychosis? An emerging hypothesis of insular dysfunction. J Psychiatry Neurosci 2012; 37: 17-27.

18 Etkin A, Prater KE, Schatzberg AF, Menon V, Greicius MD. Disrupted amygdalar subregion functional connectivity and evidence of a compensatory network in generalized anxiety disorder. Arch Gen Psychiatry 2009; 66 : 1361-72.

19 Spitzer RL, Williams JB, Gibbon M, First MB. The Structured Clinical Interview for DSM-III-R (SCID). I: history, rationale, and description. Arch Gen Psychiatry 1992; 49: 624-9.

20 American Psychiatric Association. Diagnostic and Statistical Manual of Mental Disorder (4th edn) (DSM-IV). APA, 1994.

21 Kay SR, Fiszbein A, Opler LA. The positive and negative syndrome scale (PANSS) for schizophrenia. Schizophr Bull 1987; 13: 261-76.

22 Murphy K, Bodurka J, Bandettini PA. How long to scan? The relationship between $\mathrm{fMRI}$ temporal signal to noise ratio and necessary scan duration. Neuroimage 2007; 34: 565-74.

23 Van Dijk KR, Sabuncu MR, Buckner RL. The influence of head motion on intrinsic functional connectivity MRI. Neuroimage 2012; 59: 431-8.

24 Martinez D, Slifstein M, Broft A, Mawlawi O, Hwang DR, Huang Y, et al. Imaging human mesolimbic dopamine transmission with positron emission tomography. Part II: amphetamine-induced dopamine release in the functional subdivisions of the striatum. J Cereb Blood Flow Metab 2003; 23: 285-300.

25 Birn RM, Murphy K, Bandettini PA. The effect of respiration variations on independent component analysis results of resting state functional connectivity. Hum Brain Mapp 2008; 29: 740-50.

26 Woods SW. Chlorpromazine equivalent doses for the newer atypical antipsychotics. J Clin Psychiatry 2003; 64: 663-7. 
27 Dandash O, Fornito A, Lee J, Keefe RS, Chee MW, Adcock RA, et al. Altered striatal functional connectivity in subjects with an at-risk mental state for psychosis. Schizophr Bull 2014; 40: 903-14.

28 Orliac F, Naveau M, Joliot M, Delcroix N, Razafimandimby A, Brazo P, et al. Links among resting-state default-mode network, salience network, and symptomatology in schizophrenia. Schizophr Res 2013; 148: 74-80.

29 Manoliu A, Meng C, Brandl F, Doll A, Tahmasian M, Scherr M, et al. Insular dysfunction within the salience network is associated with severity of symptoms and aberrant inter-network connectivity in major depressive disorder. Front Hum Neurosci 2013; 7: 930.

30 Craig AD. How do you feel? Interoception: the sense of the physiological condition of the body. Nat Rev Neurosci 2002; 3: 655-66.

31 Fletcher PC, Frith CD. Perceiving is believing: a Bayesian approach to explaining the positive symptoms of schizophrenia. Nat Rev Neurosci 2009; 10: $48-58$.

32 Corlett PR, Taylor JR, Wang XJ, Fletcher PC, Krystal JH. Toward a neurobiology of delusions. Prog Neurobiol 2010; 92: 345-69.

33 Palaniyappan L, White TP, Liddle PF. The concept of salience network dysfunction in schizophrenia: from neuroimaging observations to therapeutic opportunities. Curr Top Med Chem 2012; 12: 2324-38.

34 Kapur S. Psychosis as a state of aberrant salience: a framework linking biology, phenomenology, and pharmacology in schizophrenia. Am J Psychiatry 2003; 160: 13-23.
35 Kapur S, Mizrahi R, Li M. From dopamine to salience to psychosis - linking biology, pharmacology and phenomenology of psychosis. Schizophr Res 2005; 79: 59-68.

36 Murray GK, Corlett PR, Clark L, Pessiglione M, Blackwell AD, Honey G, et al. Substantia nigra/ventral tegmental reward prediction error disruption in psychosis. Mol Psychiatry 2008; 13: 67-76

37 Palaniyappan L, Simmonite M, White TP, Liddle EB, Liddle PF. Neural primacy of the salience processing system in schizophrenia. Neuron 2013; 79: 814-28.

38 Cole DM, Oei NY, Soeter RP, Both S, van Gerven JM, Rombouts SA, et al. Dopamine-dependent architecture of cortico-subcortical network connectivity. Cereb Cortex 2013; 23: 1509-16.

39 Lui S, Li T, Deng W, Jiang L, Wu Q, Tang H, et al. Short-term effects of antipsychotic treatment on cerebral function in drug-naive first-episode schizophrenia revealed by "resting state" functional magnetic resonance imaging. Arch Gen Psychiatry 2010; 67: 783-92.

40 Sambataro F, Blasi G, Fazio L, Caforio G, Taurisano P, Romano R, et al. Treatment with olanzapine is associated with modulation of the default mode network in patients with schizophrenia. Neuropsychopharmacology 2010; 35: 904-12. 